\title{
Integrated Memory for Object, Place, and Context in Rats: A Possible Model of Episodic-Like Memory?
}

\author{
Madeline J. Eacott and Gillian Norman \\ Department of Psychology, University of Durham Science Laboratories, Durham, DH1 3LE, United Kingdom
}

We report an investigation into memory for an object, its spatial location, and the context in which it appeared in rats. A novel task based on the spontaneous recognition paradigm is presented that requires memory for these three aspects. This task is performed easily by intact rats at delays of up to $1 \mathrm{hr}$. However, performance of the task is severely and selectively disrupted by transection of the fornix. Ability to perform this task is discussed in relation to models of episodic memory in animals, and we suggest that this task provides a simple and useful animal model of episodic memory for use with rats.

Key words: rat; episodic; memory; fornix; context; object; place

\section{Introduction}

Unambiguously demonstrating episodic memory in nonhuman animals has proved to be problematic. Tulving (1983) defined episodic memory as memory that "receives and stores information about temporally dated episodes or events and temporalspatial relations between them." Thus, one approach is to investigate memory for objects (what), their spatial location (where), and a temporal element (when). Clayton and colleagues (Clayton and Dickinson, 1998; Clayton et al., 2001) have shown that some birds are indeed able to demonstrate memory for this critical triad of memory attributes ("what, where, when"). The foodstoring habits of scrub jays were used to show that the type of food (what), its location (where), and how long ago storage took place (when) can all be remembered and used for successful cache retrieval. Clayton et al. (2001) have claimed that this ability provides a useful animal model of episodic-like memory. However, this paradigm uses hand-reared jays and relies on their natural food-storing habits, so it cannot be used directly with other common laboratory animals.

The when element of the episodic-like triad has proved to be particularly problematic in developing a task for use with rats. Thus, we have taken another approach and considered the role of context in such tasks. Memory for the object and the context in which it was found is impaired after lesions within the putative memory system in both monkeys (Gaffan and Harrison, 1989; Gaffan, 1994a,b; Gaffan and Parker, 1996; Parker and Gaffan, 1997a,b) and rats (Simpson et al., 1988; Gaffan et al., 2001). Background scenes defined the contexts, so Gaffan (1994a) used the term "scene memory" and argued that scene memory is analogous to episodic memory.

Received June 17, 2003; revised Dec. 8, 2003; accepted Dec. 17, 2003

This work was supported by Biotechnology and Biological Sciences Research Council Grant 509835 to M.J.E. and E. A. Gaffan. We thank Steve Nagle and Christine Richardson for technical assistance.

Correspondence should be addressed to Dr. M. J. Eacott, Department of Psychology, Science Laboratories, South Road, Durham, DH1 3LE, UK. E-mail: M.J.Eacott@durham.ac.uk.

DOI:10.1523/JNEUROSCI.2975-03.2004

Copyright $\odot 2004$ Society for Neuroscience $\quad$ 0270-6474/04/241948-06\$15.00/0
Although scene memory provides a possible model of episodic memory for use with nonhuman animals, it involves lengthy pretraining to demonstrate memory in both rats and monkeys. Therefore, we developed a simple and novel task to examine rats' ability to demonstrate memory for objects, their spatial position, and the context in which they appeared. It is based on the spontaneous object recognition task (Ennaceur and Delacour, 1988), which uses rats' spontaneous tendency to explore novel aspects of their environment. Rats are presented with two familiar objects, only one of which has not been encountered previously in the current location and context, although both location and context are themselves familiar. Thus, the configuration of object, location, and context is novel, although no other aspect or combination of aspects is novel. If rats possess integrated memory for the "what, where, context" triad, we predict that they will preferentially explore the object that appears in a novel configuration of place and context over an object that is in a familiar location and context.

In experiment 1 , intact animals were used to establish whether rats could reliably demonstrate memory in this task. Subsequently, in experiments 2 and 3 , the contributions of the hippocampal system (through fornix transection) and two cortical areas (through lesions of the perirhinal and postrhinal cortices) to performance in this task were examined.

\section{Materials and Methods \\ Experiment 1 \\ Subjects}

Ten experimentally naive dark Agouti rats were used. Testing began when the animals were $\sim 4$ months old. They were housed in groups in diurnal conditions ( $12 \mathrm{hr}$ light/dark cycle). All testing took place during the light phase. Throughout the study, all animals had ad libitum access to food and water.

\section{Apparatus}

Testing was conducted in an open field made of wood (base dimensions, $1 \mathrm{~m}^{2}$; height, $48 \mathrm{~cm}$ ). It could be configured to provide two different contexts. In context 1 , the base was painted matte black, and the walls were painted matte white. In context 2, the base was $1.5 \mathrm{~cm}^{2}$ white 

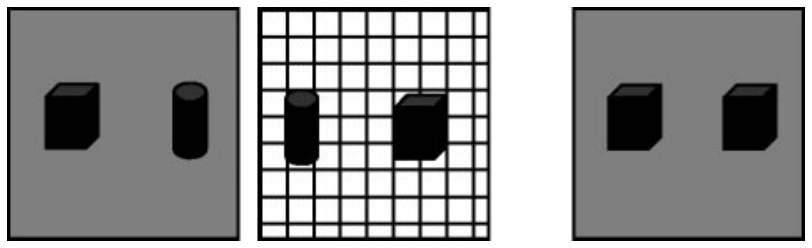

Figure 1. Aschematic representation of an example of two exposure phases and a test phase from experiments 1 and 2 . In the two exposure phases, the open field contains two different objects, whereas in the test phase, two copies of a single object are used.

plastic-coated wire mesh overlaid on a black base, and the walls were natural wood. Junk objects were placed into the open field equidistant from the sides of the open field. The objects used were gathered from a range of sources, with the following collection criteria: it should be possible to collect four identical copies; they should be stable (or suitable for weighting) so that they do not topple when explored by rats; they should all be of a broadly similar size; and they should be made of a material that cannot be gnawed easily by the rats within the confines of the experiment (e.g., glass, metal). Examples of objects used include bottles, jars, candlesticks, and ornaments.

\section{Habituation}

The rats received eight habituation sessions at a rate of one per day, to habituate them to the open field, to the contexts to be used in the study, and to the junk objects. Four habituation sessions used context 1, and four used context 2 . In each habituation session, a different single object was placed in the center of the open field. These objects were not reused in later parts of this study. During habituation, the rats were placed in the open field and allowed to explore freely. The first habituation session in each context took place in cage-mate groups and lasted $30 \mathrm{~min}$. Subsequent sessions took place singly, and each lasted $10 \mathrm{~min}$. After completion of habituation, testing of the main task took place.

\section{Behavioral testing}

In the experimental task, each trial consisted of two exposure phases followed at a delay of $2,5,10,15,30,60$, or $120 \mathrm{~min}$ by a test phase (Fig. $1)$. In the first exposure phase, the open field was configured as context 1 and contained a copy of a novel object (A) on the left side of the open field and a copy of a different novel object (B) on the right side. For the second exposure phase, the open field was configured as context 2 and contained a second copy of object B on the left side and a second copy of object A on the right side. In the test phase, the open field was configured either as context 1 or as context 2 and contained two additional copies of either object $A$ or object $B$, one on the left and one on the right, equidistant from the sides of the open field. Multiple copies of objects were used to prevent odor marking, etc.

For each exposure phase, the animal was placed into the open field and allowed to explore until it had spent at least $15 \mathrm{sec}$ exploring each object, subject to a minimum of $2 \mathrm{~min}$ and a maximum of $5 \mathrm{~min}$ in the open field. Exploration of an object was defined as the rat's nose being within $1 \mathrm{~cm}$ of and oriented toward the object, sniffing at, or otherwise closely attending to the object. This definition excludes using the object merely as support during rearing or sitting on the object. After the first exploration phase, the rat was removed from the open field and placed in a holding cage for $\sim 2 \mathrm{~min}$ to allow the objects and context within the open field to be changed. The animal was then returned to the open field for the second exploration phase. After the second exploration phase, there was a delay of $2,5,10,15,30,60$, or $120 \mathrm{~min}$ in the holding cage before the animal was returned to the open field for the test phase. In the test phase, each rat was placed in the open field for $3 \mathrm{~min}$, and the time spent exploring each of the two copies of the object was recorded.

Each rat was tested at each delay on four separate occasions, each using novel objects. On two of these occasions, context 1 was used in the test phase, and on two occasions, context 2 was used. Equally, within each context, one test phase used two copies of object A, and the other used object B. Thus, at each delay, each animal was tested using all four combinations of context and objects. The left-right position within the open field in which an object appeared in each context was controlled between animals, as was the order in which the contexts were experienced. Rats experienced only one test session (two exploration phases and one test phase) per day and were tested 5 or $6 \mathrm{~d}$ per week.

\section{Experiment 2}

Subjects

A total of 46 dark Agouti rats that had taken part previously in tests involving object-context configurations within the spontaneous recognition paradigm (Norman and Eacott, 2004) were used. Testing began $\sim 3$ weeks after completion of those experiments, when they were $\sim 8$ months old. They were housed in pairs in diurnal conditions $(12 \mathrm{hr}$ light/dark cycle) and tested during the light cycle. Because the animals had completed other experiments recently in the same apparatus, they did not require additional habituation.

\section{Surgery}

Surgery was performed on 50 animals. Four animals died during surgery or as a result of perioperative complications. Of the remainder, 12 animals received bilateral lesions of the perirhinal cortex, 12 received bilateral lesions of the postrhinal cortex, 11 animals received bilateral fornix lesion, and 11 animals were sham operated.

Perirhinal lesions. The target lesion was based on the limits of the perirhinal cortex, as delineated by Burwell and colleagues (Burwell et al., 1995; Burwell and Amaral, 1998; Burwell, 2001). Each rat was anesthetized using halothane, and then its head was shaved and positioned in a stereotaxic headholder angled at $5^{\circ}$. A total of $0.5 \mathrm{ml}$ of the analgesic vetagesic was administered subcutaneously. The scalp was incised along the midline, and bregma was measured at an angle of $12^{\circ}$. The top of the skull was measured at three points, 3,4 , and $5 \mathrm{~mm}$ posterior and $5.1 \mathrm{~mm}$ lateral to bregma. Using a dental drill, a portion of the lateral surface of the skull overlying the rhinal sulcus was removed. The dura was cut to allow the insertion of an electrode into the brain. Lesions were made using an RFG4-A RF lesion generator (Radionics, Burlington, MA). The electrode (tip length, $0.3 \mathrm{~mm}$; diameter, $0.25 \mathrm{~mm}$ ) was lowered at an angle of $12^{\circ}$ at the three points stated above to a depth of $6.6 \mathrm{~mm}$ below the top of the skull. Current was passed such that a temperature of $\sim 75^{\circ} \mathrm{C}$ was achieved for $1 \mathrm{~min}$. The electrode was then withdrawn, bregma was measured at an angle of $-12^{\circ}$, and the procedure was performed contralaterally. The scalp was closed using wound clips, and antibacterial wound powder was applied. Each animal received $5 \mathrm{ml}$ of warmed saline and $0.3 \mathrm{ml}$ of the respiratory stimulant milophyline subcutaneously.

Postrhinal lesions. The target lesion was based on the limits of the postrhinal cortex, as delineated by Burwell and colleagues (Burwell et al., 1995; Burwell and Amaral, 1998; Burwell, 2001). The initial procedure was the same as that for the perirhinal lesions, with the exception that ear bar zero was also measured. After a midline incision, both bregma and lambda were measured. Multiple estimates of the three lesion sites were obtained using measurements based on bregma, lambda, and ear bar zero using the coordinates shown in Table 1.

When estimates based on different sites differed, judgment was used, with greater weight being given when two sets of calculations agreed closely. The top of the skull was measured at each of the selected sites. An area of the skull overlying the target region was removed, the dura was cut at each site to allow the insertion of an electrode into the brain, and the electrode was lowered vertically to the required depth. Current was passed as described previously. The procedure was repeated contralaterally, and the scalp was closed.

Fornix lesions. The initial procedure was the same as that used for postrhinal lesions. Once the midline incision had been made, bregma was measured, and the first lesion site was calculated as $5.3 \mathrm{~mm}$ anterior and $0.7 \mathrm{~mm}$ lateral to ear bar zero and $0.4 \mathrm{~mm}$ posterior and $0.7 \mathrm{~mm}$ lateral to bregma. A mean was used when the anteroposterior positions differed between the two calculations. A second target site was calculated in the same way, using a mediolateral measurement of $1.7 \mathrm{~mm}$ lateral to both bregma and ear bar zero. The skull overlying these sites on each side of the midline was removed in a single section. The top of the dura was measured at each of the target sites. The dura was cut at each site, and the electrode was lowered. At the first site, depths of $4.5 \mathrm{~mm}$ relative 
Table 1. Coordinates of the lesion sites for postrhinal lesions relative to landmarks of ear bar zero (EBZ), bregma (Br), and lambda (Lmd)

\begin{tabular}{|c|c|c|c|c|c|c|c|c|c|}
\hline & \multicolumn{3}{|l|}{ Site 1} & \multicolumn{3}{|l|}{ Site 2} & \multicolumn{3}{|l|}{ Site 3} \\
\hline & EBZ & $\mathrm{Br}$ & Lmd & EBZ & $\mathrm{Br}$ & Lmd & EBZ & $\mathrm{Br}$ & Lmd \\
\hline AP & +0.2 & -5.8 & +2.0 & -1.3 & -7.1 & +0.7 & -1.8 & -7.6 & +0.4 \\
\hline$M L$ & \pm 6.1 & & & \pm 5.8 & & & \pm 5.7 & & \\
\hline DV & +2.8 & -8.5 & -6.6 & +3.8 & -7.8 & -5.8 & +4.2 & -6.8 & -4.8 \\
\hline
\end{tabular}

All measurements are given in millimeters. AP, Anteroposterior; ML, mediolateral; DV, dorsoventral.
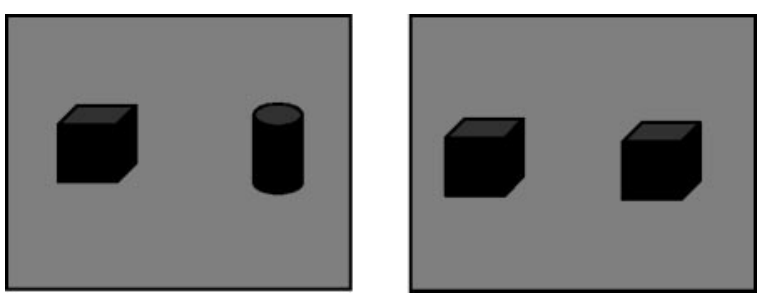

Figure 2. A schematic representation of an example of an exposure and a test phase from experiment 3. In the exposure phase, the open field contains two different objects, whereas in the test phase, two copies of a single object are used.

to the top of the skull measured at bregma and $3.7 \mathrm{~mm}$ relative to the top of the dura were used. At the second site, the depths were $4.6 \mathrm{~mm}$ relative to the top of the skull at bregma and $3.8 \mathrm{~mm}$ relative to the top of the dura. Current was passed as described previously. The procedure was repeated on the contralateral side, and then the scalp was closed.

Sham surgery. The procedure for the sham animals was identical to that for the animals in the lesion groups, except that the electrode was not lowered into the brain. Four of the animals had their skulls removed as if for a perirhinal lesion, four had their skulls removed as if for a bilateral postrhinal lesion, and three had their skulls removed as if for a bilateral fornix lesion.

For histological purposes, at the end of testing, operated animals were perfused intracardially with a $5 \%$ formal saline solution. Their brains were removed, embedded in wax, and coronally sectioned into $10 \mu \mathrm{m}$ slices. Every 10th section was stained with cresyl violet (Nissl stain).

\section{Behavioral task}

The procedure was identical to that for experiment 1 , except that only delays of 2 and 5 min were used.

\section{Experiment 3: memory for objects and place}

Experiment 3 was designed as a control for the effect of place alone on the familiarity of an object in experiment 2 . The same animals used in experiment 2 took part in experiment 3 . The open field was configured as context 1 throughout this experiment. There was a single exposure phase that was identical to the first exposure phase of experiment 1 (i.e., object A on the left and object B on the right) (Fig. 2). After the single exposure phase and a delay of 2 or $5 \mathrm{~min}$, the animal was returned to the open field for the test phase. For the test phase, the open field contained two copies of object A, one on the left and one on the right. The test phase lasted 3 min, and the time spent exploring each of the two copies of object A was recorded. The side of the open field on which each object appeared in the exposure phase was controlled for between animals, as was the object used in the test phase. The experiment was repeated twice at each delay using different objects each time.

\section{Data analysis}

The difference between time spent exploring the objects in the novel and familiar object-place context (experiments 1 and 2) or object-place (experiment 3) combinations was calculated as a proportion of the total time spent exploring both objects (Ennaceur and Delacour, 1988). On this measure, a value of zero reveals no difference in exploration of the two objects. Values higher than zero (with a maximum of one) reveal greater exploration of the novel combination of object-place context (experiments 1 and 2) or object-place (experiment 3 ).

\section{Results \\ Histology \\ Perirhinal}

One animal died before perfusion could take place. However, death occurred a substantial amount of time after the completion of testing. There was no indication that this animal's performance was in any way affected by illness or disability. Histological analysis revealed that in all other cases, the perirhinal lesions were essentially as intended, extending $\sim 3.0-6.5 \mathrm{~mm}$ posterior to bregma, as delineated by Burwell et al. (1995), although they were not complete lesions, with some sparing of the caudal part of the perirhinal cortex. This was significant in five of the animals, although lesion size assessed qualitatively showed no relationship with performance. Figure 3 (left) shows the extent of the smallest and largest lesions.

\section{Postrhinal}

As intended, the lesions were all posterior to those found in the animals with perirhinal lesions. There was considerable variation in the extent of the lesions, but they were all within the area delineated by Burwell and colleagues (Burwell et al., 1995; Burwell and Amaral, 1998; Burwell, 2001), although a number of animals showed some damage to the temporal cortex. Figure 3 (middle) shows the extent of the largest and smallest lesions. Qualitative analysis showed no indication that the size of these lesions affected behavioral performance

Fornix

All of the animals had extensive bilateral damage to the fornix. In all cases, the fornix was transected completely at anterior levels, although the anteroposterior extent of the damage varied. All animals had bilateral damage to the posterior part of the lateral septum in addition to the fornix lesions. There was some hippocampal damage in those animals with more extensive lesions, with two animals showing bilateral damage and three showing unilateral damage. There was no damage to the corpus callosum. Figure 3 (right) shows the extent of the largest and smallest lesions.

\section{Behavioral}

Experiment 1: memory for objects, place, and context in intact rats The data were analyzed using a 7 (delay) $\times 2$ (context) ANOVA, which revealed a large effect of delay $(F=4.23$; $\mathrm{df}=6,54 ; p=$ $0.001)$ but no effect of context $(F<1)$ and no interaction between delay and context $(F<1)$. Analysis at each delay using onesample $t$ tests showed that, as can be seen in Figure 4, animals differed from chance (zero) at all delays except $2 \mathrm{hr}(2-15 \mathrm{~min}$, $t>3.9, \mathrm{df}=9, p<0.01 ; 30 \mathrm{~min}$ and $1 \mathrm{hr}, t>2.60, \mathrm{df}=9, p<$ $0.05 ; 2 \mathrm{hr}, t<1)$.

In total exploration, there was a main effect of delay $(F=3.18$; $\mathrm{df}=6,54 ; p=0.01)$, and although there was no effect of context $(F=1.61 ; \mathrm{df}=1,9 ; p>0.05)$, there was an interaction between delay and context $(F=6.54 ; \mathrm{df}=6,54 ; p<0.001)$. These effects were caused by the animals showing less exploration in context 1 than in context 2 at delays of 5 and $10 \mathrm{~min}$. 

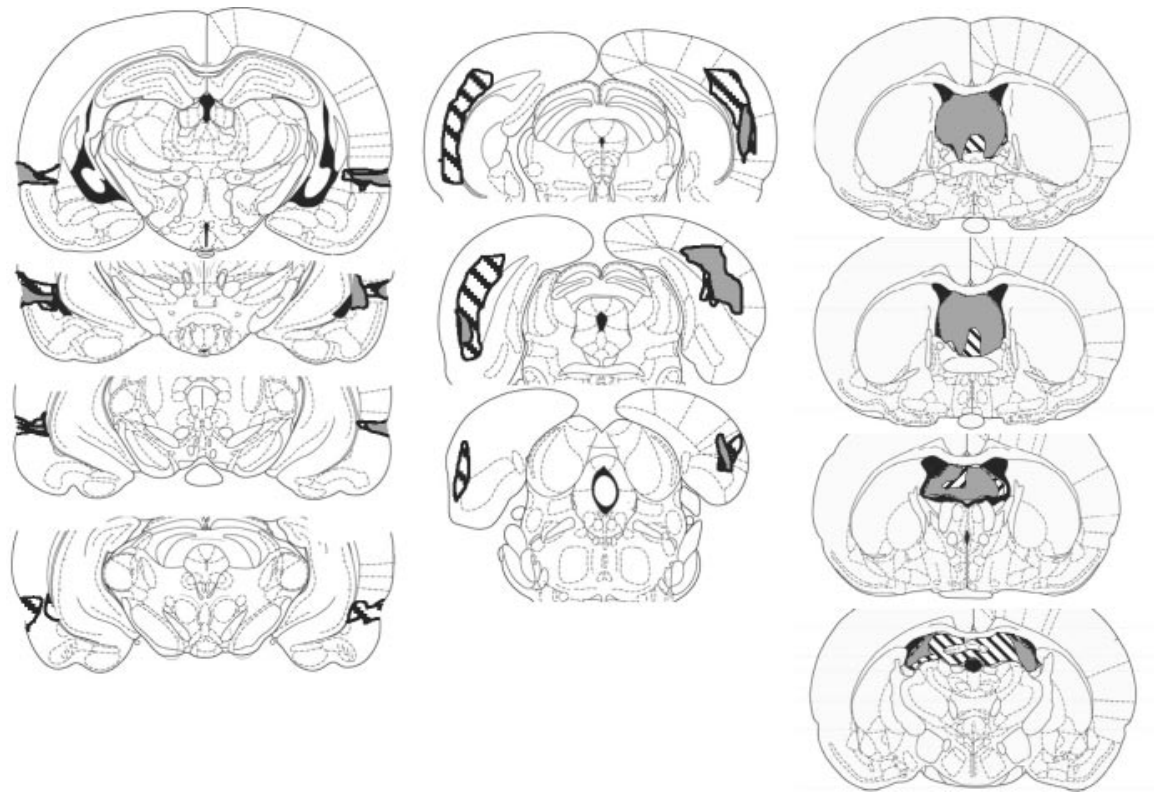

Figure 3. Histological results for the three lesion groups. From left to right, the groups are perirhinal, postrhinal, and fornix. On each section, the largest (stripes) and smallest (semitransparent gray) lesions are shown on standard sections taken from the atlas of Paxinos and Watson (1998).

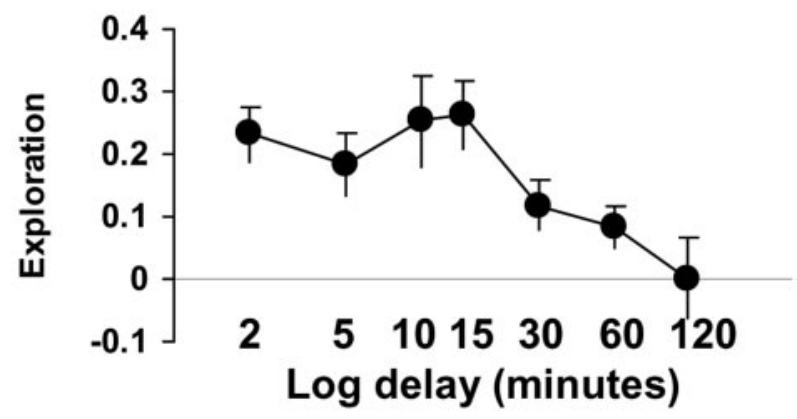

Figure 4. The results from experiment 1 , as the mean difference between time spent exploring the objects in the novel and familiar object-place-context combinations, calculated as a proportion of the total time spent exploring both objects. On this measure, a value of zero reveals no difference in exploration of the two objects. Values higher than zero reveal greater exploration of the novel object-place- context combination. Errors bars represent SEM.

Experiment 2: memory for objects, place, and context A 4 (lesion) $\times 2$ (context) $\times 2$ (delay) ANOVA revealed a large difference between the groups $(F=17.58 ; \mathrm{df}=3,40 ; p<0.001)$. Post hoc analysis of these main effects of lesion using Tukey's test showed that the fornix animals were severely impaired compared with the sham animals $(p<0.001)$ (Fig. 5). They were also severely impaired compared with both perirhinal and postrhinal animals $(p<0.001)$, although no other lesion group differed from the shams. There were no effects of delay $(F=2.00 ; \mathrm{df}=1$, $40 ; p>0.05)$ or of context $(F<1)$ and no interactions between the variables $(F<1)$, indicating that the fornix deficit was consistent across delays and testing contexts. One-sample $t$ tests at each delay indicated that all groups were above chance at both delays (in all cases, $t>5 ; p<0.001$ ) except the fornix-lesioned animals (in both cases, $t<1$ ). (Degrees of freedom are 10 for sham and fornix groups and 11 for perirhinal and postrhinal groups.) Thus, there is no evidence that the behavior of the fornix group discriminated between the objects in familiar and novel combinations of object-place and context.

Analysis of the levels of object exploration shown by the groups using a 4 (lesion) $\times 2$ (context) $\times 2$ (delay) ANOVA showed that there was no difference between the groups in the total amount of object exploration $(F=1.08$; $\mathrm{df}=3,40 ; p>0.05)$, no effect of delay on the amount of exploration $(F=1.48$; $\mathrm{df}=$ $1,40 ; p>0.05)$, and no interaction between delay and lesion $(F<1)$. Thus, there is no evidence that the differences shown above can be attributed to differences between groups in levels of exploration.

Experiment 3: memory for objects and place

This experiment was designed to control for the effect of place in experiment 1. A 4 (lesion) $\times 2$ (delay) ANOVA was performed (Fig. 6), which revealed no differences between the groups $(F<1)$. There was also no effect of delay $(F<1)$ and no interaction $(F<1)$. One-sample $t$ tests showed that all groups were above chance at both delays $(t>3.27 ; p<0.01)$ except for the perirhinal animals at $2 \mathrm{~min}(t=$ 2.03; $p=0.07)$. However, because this group was above chance at $5 \mathrm{~min}(t=3.29$; $p<0.01)$, this result is anomalous. Degrees of freedom are 10 for sham and fornix groups and 11 for perirhinal and postrhinal groups. There was no difference between the groups in total exploration $(F=2.640$; $\mathrm{df}=3,40 ; p=$ $0.063)$, but there was a main effect of delay $(F=8.91$; $\mathrm{df}=1,40$; $p<0.01)$ and an interaction between delay and lesion $(F=3.40$; $\mathrm{df}=3,40 ; p<0.05)$. This resulted from all groups except the fornix animals showing less exploration at the 5 min delay than at the 2 min delay, with the fornix animals displaying the opposite pattern of behavior.

\section{Discussion}

Both intact and sham-operated rats showed a clear preference for exploring an object that is in a novel configuration with location and context over an object that is in a familiar configuration. Although this preference diminished with increasing delay, in intact rats, it was robust at delays of up to $15 \mathrm{~min}$ and apparent at delays of up to $1 \mathrm{hr}$. Moreover, the memory was formed and expressed in the absence of explicit training or motivation and thus reflects a naturally formed memory.

Memory over a $1 \mathrm{hr}$ delay in this task can be contrasted with memory within the standard spontaneous recognition paradigm, for which sham-operated rats in our laboratory have shown memory for single objects at delays of at least $24 \mathrm{hr}$ (Norman and Eacott, 2004). Although memory in the current task is apparent only at delays of up to $1 \mathrm{hr}$, the task used two different presentation episodes, and because either context could be used at the test phase, memory for both episodes was demonstrated. Moreover, the two presentation episodes were very similar, yet the memory contained sufficient detail to distinguish between them. Studies using a range of objects in the standard spontaneous recognition paradigm in our laboratory have shown that the delay over which reliable discrimination can be shown is much reduced when the stimuli are more confusable (Norman and Eacott, 2004). Thus, a direct comparison with simple object recognition in the standard spontaneous recognition paradigm is not valid. In the present task, rats showed memory for two different, highly confusable 


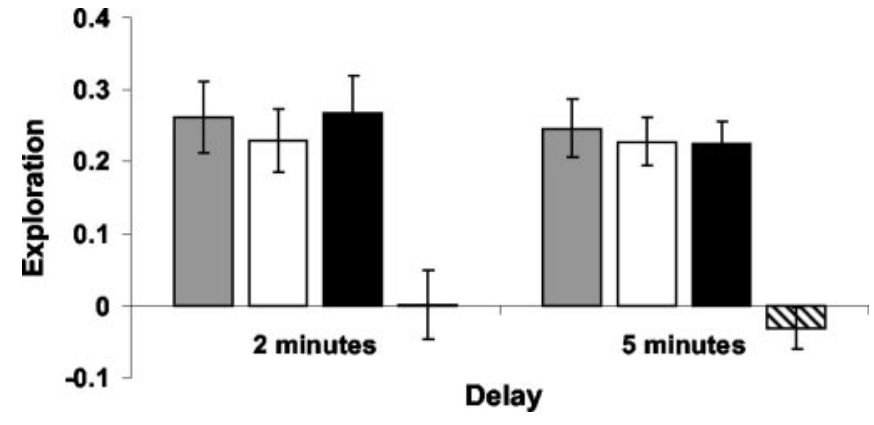

Figure 5. The results from experiment 2, as the mean difference between time spent exploring the objects in the novel and familiar object-place-context combinations, calculated as a proportion of the total time spent exploring both objects. On this measure, a value of zero reveals no difference in the exploration of the two objects. Values higher than zero reveal greater exploration of the novel object-place-context combination. Errors bars represent SEM.

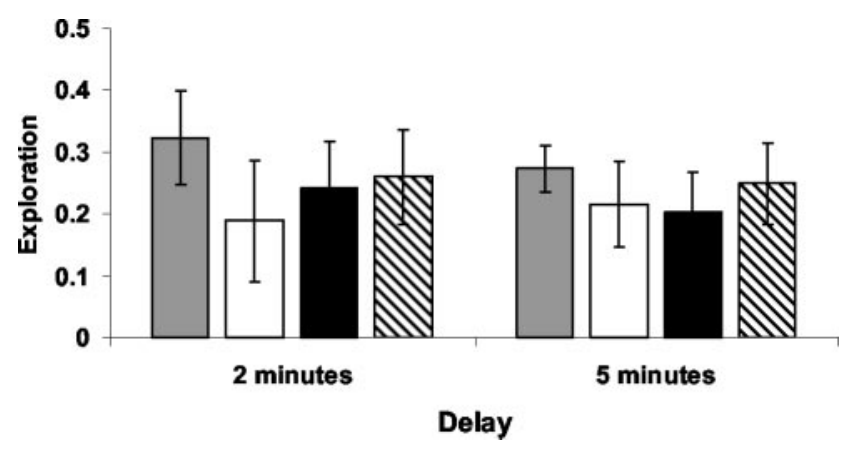

Figure 6. The results from experiment 3 as the mean difference between time spent exploring the objects in the novel and familiar object-place combinations, calculated as a proportion of the total time spent exploring both objects. On this measure, a value of zero reveals no difference in exploration of the two objects. Values higher than zero reveal greater exploration of the novel object-place combination. Errors bars represent SEM.

episodes after a single exposure over delays of up to $1 \mathrm{hr}$, which compares well with other memory tasks.

Having established that the rats can demonstrate relatively long-lasting memory for configurations of objects, their spatial locations, and the contexts in which they appeared, we consider further the nature of the memory. In this task, two highly similar presentation events were distinguished by the contexts in which they appeared. Thus, context served as an "occasion specifier," which was used in memory to distinguish the two occasions. We argue that this is similar to the role played by temporal context in the demonstration of episodic-like memory in scrub jays (Clayton and Dickinson, 1998; Clayton et al., 2001). The temporal aspect in these studies may be viewed as merely defining the temporal context of the caching event, aiding in distinguishing one event from other similar events. In humans, memory for the temporal context of events is notoriously poor and is dissociable from episodic memory itself (for review, see Friedman, 1993). Thus, although episodic memory in humans is associated with a feeling of the past (Tulving, 2001), it need not involve memory for the time of occurrence, although it does involve memory for events that occurred on a specific past occasion. Thus, we argue that the temporal aspect of the episodic triad (when) may be merely a particular example of a characteristic of the memory that specifies the occasion of the event. We argue that it is in this occasion-specification role that temporal context may be important to episodic-like memory. This role may be represented equally by context, as it is in the current study. Thus, we argue that the critical triad of episodic-like memory may be redefined to include any occasion-specifying characteristic of event memory in place of the specifically temporal when. In this sense, both the episodic-like memory exhibited by jays (Clayton and Dickinson, 1998; Clayton et al., 2001) and scene memory (Gaffan, 1994a) involve our redefined triad of episodic-like memory (what, where, and occasion-specifying context).

To assess the significance of rats' ability in this task, we also consider whether the performance demonstrated could occur on the basis of familiarity alone (Mandler, 1980). In this task, all of the components (objects, locations, and contexts) are equally familiar. Moreover, even combinations of any two components are equally familiar. For example, object A has been experienced in both context 1 and 2 and has appeared on both the left and the right. It is only the combination of object, context, and location that is novel. This rules out some possible explanations of our results. For example, one cannot claim that an object appears relatively novel because the reflective qualities of the context give it a slightly different appearance: both objects have been experienced in both contexts. One would have to claim that the unique combination of object position and context resulted in a subtly different appearance sufficient to result in a strong preference in an untrained rat. We do not consider that this explanation of our results is plausible. However, we are currently devising experiments to exclude conclusively this possibility.

If the current task were a model of episodic memory, it would be predicted that memory in this task should be severely impaired by lesions within the hippocampal system. This is indeed the case. Fornix transection resulted in a severe impairment in this task, an effect specific to fornix lesions, because neither perirhinal nor postrhinal lesions impaired the task at the delays tested.

The lack of impairment in the perirhinal group is not surprising: although causing clear impairments of object recognition at longer delays than are used here (Ennaceur et al., 1996), perirhinal lesions do not impair object-place memory (Ennaceur et al., 1997) and cause relatively mild, delay-dependent impairments of object-context memory (Norman and Eacott, 2004). However, we found previously that postrhinal lesions impair memory for object-context associations to a greater extent than do fornix lesions (Norman and Eacott, 2004), suggesting that the present task makes different demands on memory than the "object-in-context" task. Indeed, this result was found with the very same group of postrhinal animals. Thus, the absence of an impairment in the current study cannot be ascribed to the absence of a functional lesion in the postrhinal groups. Equally, the perirhinal group has revealed previously a marked impairment in object recognition (Norman and Eacott, 2004), again confirming that the lesion was functional.

It initially appeared possible that the fornix deficit in the object-place context task resulted from the object-place element, because fornix animals have been found repeatedly to be impaired on such a task (Ennaceur and Meliani, 1992; Ennaceur et al., 1997). Yet we found no object-in-place deficit (experiment 3 ). However, the delays used here are extremely short compared with the standard 15 min delay used by many studies, so that the failure to find a deficit in any of the groups is less surprising than it might seem at first. An impairment at longer delays might reasonably be predicted. Nonetheless, the lack of an impairment in the object-place task at the delays used contrasts strongly with the severe fornix impairment in the object-place-context task at even very short delays, and thus emphasizes the importance of the fornix for the object-place-context task.

Our design has some similarity to object-in-context designs 
(Dix and Aggleton, 1999). However, neither hippocampal lesions (Mumby et al., 2002) nor fornix lesions (Norman and Eacott, 2004) result in impairments in object-in-context tasks, which are as uniform or as severe as those reported here. However, the more severe deficits reported here do not result from increased task difficulty, because our task was acquired effortlessly by shamoperated animals. Therefore, it must be concluded that the fornix impairment is produced by the combination of object, place, and context rather than by any of these elements alone or in pairs or by the difficulty of the task itself.

These results suggest that the current object-place-context task is processed by a different memory system than that involved in processing either the object-in-context (Mumby et al., 2002) or the standard spontaneous object recognition (Ennaceur et al., 1997) tasks. Such a memory system would appear to depend on an intact hippocampus, either alone or as part of an extended memory system concerned with episodic-like memory. In contrast, the standard object recognition task is heavily dependent on the perirhinal cortex (Ennaceur et al., 1996), whereas the objectin-context task is dependent on an intact postrhinal cortex (Norman and Eacott, 2004). This may reflect the difference between the recall of an episodic memory relating to a specific event defined by occasion and place compared with mere familiarity (Aggleton and Brown, 1999), which may be sufficient for adequate performance on the object-in-context task or the standard spontaneous object recognition task. Under such an account, the more restricted impairments found in studies of object-context memory with fornix (Norman and Eacott, 2004) or hippocampal (Mumby et al., 2002) lesions reflect the fact that an episodic-like memory system may contribute but is not critical to the objectin-context discrimination.

In summary, we present a novel task involving discrimination of object, place, and context combinations. Like episodic memory in humans, memory in this task is acquired easily without apparent effort or motivation and is selectively impaired by disruption of the hippocampal system. By arguing that context performs an occasion-specifying role that may be similar to the temporal aspect normally viewed as being essential to episodic-like memory, we believe that this task may provide a simple and effective model of episodic-like memory for the rat.

\section{References}

Aggleton JP, Brown MW (1999) Episodic memory, amnesia, and the hippocampal-anterior thalamic axis. Behav Brain Sci 22:425-489.

Burwell RD (2001) Borders and cytoarchitecture of the perirhinal and postrhinal cortices in the rat. J Comp Neurol 437:17-41.

Burwell RD, Amaral DG (1998) Perirhinal and postrhinal cortices of the rat: interconnectivity and connections with the entorhinal cortex. J Comp Neurol 391:293-321.

Burwell RD, Witter MP, Amaral DG (1995) The perirhinal and postrhinal cortices of the rat: a review of the neuroanatomical literature and comparison with findings from the monkey brain. Hippocampus 5:390-408.
Clayton NS, Dickinson A (1998) Episodic-like memory during cache recovery by scrub jays. Nature 395:272-274.

Clayton NS, Griffiths DP, Emery NJ, Dickinson A (2001) Elements of episodic-like memory in animals. Philos Trans R Soc Lond B Biol Sci 356:1483-1491.

Dix SL, Aggleton JP (1999) Extending the spontaneous preference test of recognition: evidence of object-location and object-context recognition. Behav Brain Res 99:191-200.

Ennaceur A, Delacour J (1988) A new one-trial test for neurobiological studies of memory in rats. I. Behavioral data. Behav Brain Res 31:47-59.

Ennaceur A, Meliani K (1992) A new one-trial test for neurobiological studies of memory in rats. III. Spatial vs nonspatial working memory. Behav Brain Res 51:83-92.

Ennaceur A, Neave N, Aggleton JP (1996) Neurotoxic lesions of the perirhinal cortex do not mimic the behavioural effects of fornix transection in the rat Behav Brain Res 80:9-25.

Ennaceur A, Neave N, Aggleton JP (1997) Spontaneous object recognition and object location memory in rats: the effects of lesions in the cingulate cortices, the medial prefrontal cortex, the cingulum bundle and the fornix. Exp Brain Res 113:509-519.

Friedman WJ (1993) Memory for the time of past events. Psychol Bull 113:44-66.

Gaffan D (1994a) Scene-specific memory for objects: a model of episodic memory impairment in monkeys with fornix transection. J Cogn Neurosci 6:305-320.

Gaffan D (1994b) Dissociated effects of perirhinal cortex ablation, fornix transection and amygdalectomy: evidence for multiple memory systems in the primate temporal lobe. Exp Brain Res 99:411-422.

Gaffan D, Harrison S (1989) Place memory and scene memory: effects of fornix transection in the monkey. Exp Brain Res 74:202-212.

Gaffan D, Parker A (1996) Interaction of perirhinal cortex with the fornix fimbria: memory for objects and object in place memory. J Neurosci 16:5864-5869.

Gaffan EA, Bannerman DM, Warburton EC, Aggleton JP (2001) Rats' processing of visual scenes: effects of lesions to fornix, anterior thalamus, mammillary nuclei or the retrohippocampal region. Behav Brain Res 121:103-117.

Mandler G (1980) Recognizing: the judgment of previous occurrence. Psychol Rev 97:252-271.

Mumby DG, Gaskin S, Glenn MJ, Schramek TE, Lehmann H (2002) Hippocampal damage and exploratory preferences in rats: memory for objects, places, and contexts. Learn Mem 9:49-57.

Norman G, Eacott MJ (2004) Impaired object recognition with increasing levels of feature ambiguity in rats with perirhinal cortex lesions. Behav Brain Res 148:79-91.

Parker A, Gaffan D (1997a) The effect of anterior thalamic and cingulate cortex lesions on object-in-place memory in monkeys. Neuropsychologia 35:1093-1102.

Parker A, Gaffan D (1997b) Mammillary body lesions in monkeys impair object-in-place memory: functional unity of the fornix-mammillary system. J Cogn Neurosci 9:512-521.

Paxinos G, Watson W (1998) The rat brain in stereotaxic coordinates. London: Academic.

Simpson EL, Gaffan EA, Eacott MJ (1988) Rats' object-in-place encoding and the effect of fornix transection. Psychobiology 26:190-204.

Tulving E (1983) Elements of episodic memory, pp 385. Oxford: Clarendon.

Tulving E (2001) Episodic memory and common sense: how far apart? In: Episodic memory. New directions in research (Baddeley A, Conway C, Aggleton JP, eds), pp 269-287. London: Oxford UP. 